\title{
XIX.
}

Aus der medicinischen Klinik des Prof. Kraus in Graz.

\section{Ueber den Kohlenstoffgehalt des Harnes fiebernder Menschen und sein Verhältniss zur Stickstoffausscheidung.}

Von

Dr. Wilhelm Schoiz.

Ueber das Verhalten des Kohlenstoffgehaltes des Harnes fiebernder Thiere und Menschen liegen bisher nur sebr wenig Angaben vor. Die ältere Beobachtung Ewald's ${ }^{1 *}$ ), dass der Harn fiebernder Menschen reicher ist an freier Kohlensäure als der normale, konomt hier wohl kaum in Betracht. A. Loe w $\mathrm{y}^{2}$ ) injicirte Hunden $5-6 \mathrm{ccm}$ Höllensteinlösung in die Lungen, worauf eine allerdings nicht sehr erhebliche Temperatursteigerung, sowie Dyspnoe eintrat. Nur in einigen derartigen Versuchen bestimmte er das Verhältniss des Kohlenstoffes zum Stickstoff im Harne und fand dasselbe so geändert, dass relativ mehr Kohlenstoff als Stickstoff gegenüber der Norm ausgeschieden wird. Angaben darüber, wie der Koblenstoffgehalt bestimmt wurde, und Zahlenbelege für die erwähnte Aenderung führt A. Loewy leider keine an.

Sonst hat nur noch R. M a $y^{3}$ ), welcher in einer umfangreichen Arbeit die Kenntniss des Stoffwechsels fiebernder Thiere erweiterte, auch mehrere Untersuchungen ber den mit dem Harn ausgeschiedenen Kohlenstoff angestellt. Er suchte bei Kaninchen durch Injection von Schweinerothlaufculturen Fieber zu erzeugen. Die Thiere wurden alle 24 Stunden cathetrisirt und der Stickstoff in dem Harne theils nach Kjeldahl, theils nach Schneider-Seegen, der Kohlenstoff auf nassem Wege mittelst des von Kjeldahl modificirten Messinger'schen Verfahrens bestimmt. May tindet, dass das Ver-

*) Die kleinen Ziffern im Text beziehen sich auf das am Schlusse befindliche Literaturverzeichniss. 
hältniss $\frac{\mathrm{C}}{\mathrm{N}}$ im Fieberharn geändert ist. Der Fieberharn wird kohlenstoffreicher. Bei fünf von den Versuchsthieren wurde mittelst des bekannten, von $\mathrm{Rubne}^{4}$ ) bei (einem einzigen) Kaninchen bestimmten Quotienten $\frac{\mathrm{C}}{\mathrm{N}}(=0,7956)$ für den dritten Carenztag der Kohlenstoff blos aus dem analytisch gewonnenen Stickstoffgehalt berechnet. Die Berechtigung für die Verwendung dieses Rubner'schen Quotienten entnimmt May seinen Versuchen an zwei anderweitigen hungernden Kaninchen, bei welchen die wirklich ausgeführte Bestimmung des Kohlenstoffes am 3.-5. Hungertage relativ geringe Schwankungen dieser Relation ergeben hat. Bei einem dieser beiden Kaninchen $(G)$ bewegte sich der Coëfficient $\frac{\mathrm{C}}{\mathrm{N}}$ in der Carenzzeit (1.-4.'Tag) zwischen 0,708-0,7851, schwankte also um 9,8 Proc.; bei dem zweiten (H) am 3. und 4. Hungertage zwischen 0,81368-0,823 18, differirte also blos um 1,1 Proc. Trotzdem scheint es mir angezeigt, nur jene beiden Versuche May's zu benutzen, in welchen nicht blos der Stickstoff, sondern gleichzeitig auch der Kohlenstoff im Harn bestimmt worden ist. Denn erstlich stimmen die von May bei seinen Versuchen mit wirklich ausgeführter Kohlenstoffbestimmung gewonnenen Coëfficienten $\frac{\mathrm{C}}{\mathrm{N}}$ (3. Hungertag) zahlenmässig nicht mit dem erwähnten $\mathrm{Ku}$ b n er'schen. Die Differenz beträgt für Kaninchen $G$ - 6,98 Proc., für das Kaninchen $\mathrm{H}+3,35$ Proc., unter einander besteht also eine Abweichung von über 10 Proc. Ferner werden wir sehen, dass May bei den fiebernden Thieren nur relativ geringe Steigerungen des Kohlenstoffgehaltes nachzuweisen vermag. May schliesst aus dieser geringen Steigerung, dass der Fehler, den er durch Benutzung der Rubner'schen Zahl bei jenen Versuchsthieren, wo blos der Stickstoff und nicht gleichzeitig der Kohlenstoffgehalt quantitativ bestimmt, sondern der letztere blos in der erwähnten Weise gerechnet wurde, zu vernachlässigen sei. Ich finde hingegen, dass man um so weniger mit dem Rubner'schen Coëfficienten, der ja nicht einmal einen Mittelwerth darstellt, arbeiten kann, je geringere Kohlenstoffdifferenzen $\mathfrak{u}$ berhaupt zur Beurtheilung kommen. Endlich werden wir sehen, dass in den zwei hier in Betracht gezogenen Versuchen May's, wenn man statt der wirklichen Bestimmung die Rubner'sche Zahl zu Grunde legte, sich qualitative Aenderungen des Stoffwechsels gar nicht erschliessen lassen würden.

Die von $\mathrm{May}$ in den beiden letzterwähnten Versuchen er- 
hobenen Bestimmungsgrössen stelle ich in Tabelle I und II tibersichtlich zusammen.

TABelle I.

Kaninchen G.

\begin{tabular}{|c|c|c|c|c|c|}
\hline \multirow{2}{*}{$\begin{array}{c}\text { Versuchs- } \\
\text { tag }\end{array}$} & \multirow{2}{*}{ Temperatur } & \multicolumn{2}{|c|}{ Harn } & \multirow{2}{*}{$\frac{\mathrm{C}}{\mathrm{N}}$} & \\
\hline & & $\mathrm{C}$ in $\mathrm{g}$ & $N$ in $g$ & & \\
\hline 1. & 39,0 & 1,705 & 2,174 & 0,7851 & Carenz \\
\hline 2. & 38,5 & 1,424 & 1,874 & 0,7599 & $=$ \\
\hline 3. & $38,5-38,2$ & 1,411 & 1,907 & 0,74002 & $=$ \\
\hline 4. & $38,2-38,6$ & 1,429 & 2,019 & 0,708 & $=\quad \therefore$ \\
\hline 5. & $38,6-38,8$ & 1,529 & 2,168 & 0,7055 & Fieberanstieg \\
\hline 6. & $38,7-40,1$ & 2,030 & 2,819 & 0,7200 & Fieber \\
\hline 7. & $40,1-38,1$ & 2,050 & 2,591 & 0,7911 & Fieber \\
\hline
\end{tabular}

TABELLE II.

$\mathrm{Kaninchen} \mathrm{H}$.

\begin{tabular}{|c|c|c|c|c|c|}
\hline $\begin{array}{l}\text { Versuchs- } \\
\text { tag }\end{array}$ & $\begin{array}{c}\text { Temperatur } \\
{ }^{0} \mathrm{C} .\end{array}$ & $\mathrm{C}$ in $\mathrm{g}$ & $\mathrm{N}$ in $\mathrm{g}$ & $\frac{\mathrm{C}}{\mathrm{N}}$ & \\
\hline 1. & 39,0 & - & 1,32 & - & Carenz \\
\hline 2. & 39,0 & - & 1,14 & - & $=$ \\
\hline 3. & $39,0-39,6$ & 1,187 & 1,44 & 0,82318 & $=$ \\
\hline 4. & $39,6-39,2$ & 1,105 & 1,36 & 0,81368 & $=$ \\
\hline 5. & $39,7-41,0$ & 1,267 & 1,47 & 0,85950 & Fieberanstieg \\
\hline
\end{tabular}

Wie man erkennt, schwankt die Temperatur bei den zwei in Betracht kommenden Versuchsthieren May's vor dem Fieberversuch nicht unerheblich. Das Kaninchen $G$ hat eine zwischen 38,2 bis $39,0^{\circ}$ C. sich bewegende Temperatur, die sich im Fieber blos auf $40,1^{\circ} \mathrm{C}$. erhebt, somit blos um $0,3^{\circ} \mathrm{C}$. die obere Grenze der von Gottlie $\mathrm{b}^{5}$ ) und $\mathrm{Palmer} \mathrm{r}^{8}$ ) zwischen 38,8 bis $39,8^{0} \mathrm{C}$. verlegten Normaltemperatur des Kaninchens übersteigt. Das zweite Kaninchen $\mathrm{H}$, welches in der fieberfreien Zeit Temperaturen zwischen 39,0 bis $39,6^{\circ}$ C. aufweist, bekommt ein ziemlich starkes Fieber $(41,00$ C. $)$. Ich glaube allerdings nicht, dass auf die relativ geringe Temperatursteigerung beim ersten Versuchsthier unnöthig Gewicht gelegt werden muss; das Kaninchen befand sich jedenfalls in dem hier ausschliesslich in Betracht kommenden Infectionszustande. Der Infect ist bei beiden Thieren in einer Periode der Inanition hervorgerufen, wo die Stickstoffausscheidung in 24 Stunden annähernd im Gleichgewicht 
Ueber den Kohlenstoffgehalt des Harnes fiebernder Menschen u. s. w. 329

sich befand. Leider ist der zweite Versuch deswegen weniger verwendbar, weil es hier uiberhaupt nur einen Fiebertag gab; das Thier verendete zu schnell.

Bei dem Kaninchen G, bei welchem am fünften Carenztag eine intravenöse Injection von stark verdünnter Schweinerothlaufbouillon gemacht wurde, erfolgte an demselben Tage keine ernstliche Temperatursteigerung; erst am folgenden Tage, nach Injection einer unverdünten Cultur, erhob sich die Rectaltemperatur auf $40,1^{\circ}$, erhielt sich dann fast constant auf dieser Höhe, bis am achten Tage das Thier unter Collapserscheinungen zu Grunde ging. Sowohl der Stickstoff als auch der Kohlenstoff erwiesen sich im Fieberharne vermehrt. Die Differenz der Kohlenstoffausfuhr am fünften und sechsten Versuchstag beträgt etwa 24 Proc. Die Differenz der Quotienten für den fünften (Fieberanstieg) und siebenten Versuchstag (Fieber), nach den gewonnen analytischen Daten berechnet, macht 10,8 Proc. aus. Derselbe Quotient erfährt gegenuber der Rubner'schen Zahl aber eine Verminderung (um 0,6 Proc.). Würde May also in diesem Versuche sich blos an die Rubner'sche Zahl gehalten haben, so hätte er doch wohl aus dem Harn für das Fieber kaum eine qualitative Aenderung des Stoffwechsels erschliessen können.

Das Kaninchen $\mathrm{H}$ bekam bei einem Fieber von $1,2^{\circ} \mathrm{C}$. über die Normaltemperatur ebenfalls eine erhöhte Kohlenstoff- und Stickstoffausfuhr. Die Erhöhung der Kohlenstoffausfuhr im Urin betrug aber hier blos 12,8 Proc. gegenüber der Carenzzeit. Der Quotient $\frac{\mathrm{C}}{\mathrm{N}}$ erfährt nach Maassgabe der Bestimmungsgrössen eine Erhöhung von blos etwa 5,3 Proc. Bei Zugrundelegung der Rubner'schen Zahl hätte sich eine Differenz von etwa 7,4 Proc. ergeben. Dabei weicht die Relation $\frac{\mathrm{C}}{\mathrm{N}}$, welche May hier für den dritten Carenztag aus den Analysen rechnet, von der mehrfach erwähnten, für denselben Hungertag aufgestellten Zabl Rubner's blos um 3,35 Proc. ab. Es gilt also hier dasselbe, was vom ersten Versuchsthiere gesagt worden ist.

Wie man sieht, besteht kein Parallelismus zwischen Temperatursteigerung und der Aenderung des Quotienten $\frac{\mathrm{C}}{\mathrm{N}}$. Auch möchte ich betonen, dass die Steigerung schon beim ersten Versuchsthiere eine nur wenig hohe, beim zweiten, wo noch dazu ein einziger Fiebertag zur Verfügung steht, blos etwa 5 Proc. betragende gewesen ist. Die Grösse dieser Steigerung im Vergleiche zum Ru bn er'schen Quotienten 
ubersteigt zahlenmässig nach oben nicht die Schwankungen, welche nach May's eigenen Beobachtungen das Hungerthier unter physiologischen Bedingungen nach oben und unten darbietet. Bei beiden Versuchsthieren fehlt die Bestimmung der Ausscheidungsgrössen von Kohlenstoff und Stickstoff in der dem Fieber folgenden Zeit, da, wie erwähnt, beide Thiere verendeten. Endlich muss man doch auch in Erwägung ziehen, dass bei den in Betracht kommenden Versuchsthieren May's zwei Factoren zusammengewirkt haben, einerseits der Infect, andererseits die Verhungerung; das eine Thier war unzweifelhaft in ultimis, das zweite wenigstens schon collabirt. In einem solchen terminalen Zustande eines kranken Pflanzenfressers gewonnene Werthe werden auf den Menschen kaum obne Einschränkung tubertragen werden dürfen.

R. May hat die uns interessirenden Ergebnisse seiner Versuche (Steigerung des Kohlenstoffgehaltes des Harnes) für die von ihm aus anderweitigen Grunden aufgestellte relativ gesteigerte Kohlenhydratzersetzung im Fieber zu verwerthen gesucht. Wie May selbst nicht in Abrede stellt, unterliegt es jedoch keinem Zweifel, dass einer Steigerung des Quotienten $\frac{\mathrm{C}}{\mathrm{N}}$ im Harne auch eine anderweitige Aenderung des Stoffwechsels entsprechen kann, nämlich eine unvollständige Oxydation koblenstofthaltiger Verbindungen im Organismus. Eine solche Vermuthung liegt sogar im Fieber besonders nahe, da ja verschiedene Gründe für das Vorhandensein einer vermehrten Production und Ausscheidung von sauren $Z$ wischenstoffwechselproducten sprechen. Ferner ist gegenwärtig bereits vielfach die Einheitlichkeit des Fieberbegriffes aufgegeben, und gerade auch die Untersuchung des Stoffwechsels bei verschiedenen febrilen Infecten scheint für diese Trennung $z \mathfrak{u}$ sprechen.

Ich vermag sonach nicht zuzugeben, dass $\mathrm{M}$ a y die Frage einer Aenderung des Quotienten $\frac{\mathrm{C}}{\mathrm{N}}$ für den Harn vollkommen abschliessend beantwortet hat. Beim Menschen hat bisher die von vielen Seiten ausgeführte Untersuchung des respiratorischen Stoffwechsels eine durchgreifende qualitative Aenderung der oxydativen Umsetzungen nicht ergeben. Es hat sich vielmehr nach den übereinstimmenden Angaben der Untersucher herausgestellt, dass der respiratorische Quotient jeweilig dem Ernährungszustand der untersuchten fiebernden Patienten entsprach. Auch wenn man weiter gar keine Schwierigkeiten gegen die Verwerthung der bei Kaninchen angestellten Fieberversuche erheben will, war es sonach unbedingt nothwendig, die einschlägigen 
Verhältnisse bei Menschen mit verschiedenen febrilen Infecten zu untersuchen.

Die Anstellung solcher Versuche bei fiebernden Menschen begegnet gegenüber den vielen Vortheilen, welche sonst die Fieberuntersuchung hier für sich hat, der einen Schwierigkeit, dass man es nicht mit hungernden oder sonst im strengen Stickstoffgleichgewicht befindlichen Individuen zu thun hat. In einer frtiheren Untersuchung habe ich?) gezeigt, dass die Relation $\frac{\mathrm{C}}{\mathrm{N}}$ beim gesunden Mensehen recht bemerkenswerthe Schwankungen darbietet. Einen durchsichtigen Einfluss der Art der Ernährung vermochte ich nicht nachzuweisen. Natürlich fand ich immer ein Wachsen des ausgeschiedenen Kohlenstoffes, so oft der Stickstoffgehalt des Harnes wuchs. Bei annähernd gleicher Stickstoffexcretion im Urin bewegten sich diese Schwankungen (innerbalb weniger Tage) beispielsweise zwisehen 0,87 bis 0,95 . Die Schwankungsgrösse beträgt also bis 9,8 Proc. Damit war wenigstens ein Maassstab dafür gewonnen, wie weit eventuelle Aenderungen des combustiven Stoffwechsels tiber die normale Breite hinausgehen.

Um nun darüber bestimmten Aufschluss zu verschaffen, inwiefern der menschliche Fieberharn wirklich kohlenstoffreicher ist, und $\mathrm{ob}$ in demselben das Verbältniss des Kohlenstoffes zum Stickstoff eine Aenderung gegen die Norm erfährt, habe ich bei einer Anzahl fiebernder, beziehungsweise fiebernd gemachter Menschen den Urin während und ausserhalb (vor oder nach) der Fieberzeit untersucht. Ich erzeugte Fieber durch Tuberculininjection und untersuchte Menschen mit theils kurz, theils länger währendem, natïrlich entstandenem Fieber. Naturgemäss kam es mir mehr auf den Vergleich der Bestimmungsgrössen an, welche bei demselben Individuum gewonnen waren. Doch wurden alle Patienten unter möglichst gleichartige Bedingungen gebracht. Die Kranken mussten, falls auch fieberfreie Intervalle sich einstellten, beständig das Bett hüten. Bereits einige Tage vor Beginn der eigentlichen Versuchsperiode erhielten dieselben täglich die gleichen Nahrungsstoffe, wie während des Versuches selbst. Ich war wenigstens bemuiht, so weit es anging, auch die Nahrung qualitativ für alle Kranken gleich zu wählen. Die Quantität musste derart festgesetzt werden, dass sie für die eventuelle fieberfreie Zeit dem Patienten subjectiv eben noch genügte, und durfte nicht zu reichlich sein, so dass sie wäbrend der Fieberperiode nicht etwa verweigert wurde. Selbstrerständlich bekamen die Patienten schon kurze Zeit vor und während der Versuche keine Medicamente. 
Ich analysirte blos den Harn. Der Stickstoff wurde nach Kjeldahl-Argutinski bestimmt und das Mittel von drei gut stimmenden Proben genommen. Behufs Ermittelung des Kohlenstoffgehaltes analysirte ich den Harn mittelst einer Methode auf nassem Wege, welche ich in der schon erwähnten Abhandlung beschrieben habe. Die fortgesetzte Benutzung derselben hat mich noch weiter von ibrer Brauchbarkeit überzeugt. Ich stützte mich auch hier jedesmal auf zwei Analysen, welche genügend uibereinstimmende Resultate gaben. Der Harn wurde stets von $8 \mathrm{~h}$ frib bis zur gleichen Zeit des anderen Tages gesammelt und ein Verlust desselben bei Absetzen des Stuhles verhütet. In einigen Fällen habe ich auch, ohne dass ich darauf besonderes Gewicht legen werde, den 24 stiundigen Harn in zwei Partien gesondert gesammelt, und zwar, wenn das Fieber nur einige Stunden währte, eine Portion bis zum Eintritt der Temperaturerhöhung und die zweite während des Fiebers. Die Temperaturmessungen wurden zweistindlich in entsprechend sorgfältiger Weise vorgenommen.

\section{I.}

Zunächst führe ich zwei an vorher nicht fiebernden Tuberculösen angestellte Versuche mit Tuberculin-*)injection an. Das Mittel war frisch von Berlin bezogen und daselbst im Monat Juli hergestellt worden. Seine prompte Wirkung war vorher an zwei unzweifelhaft mit Tuberculose behafteten Individuen sichergestellt worden. Nur in einem der Versuche habe ich eine Temperatursteigerung erzielen können. Die höchste beobachtete Temperatur betrug $38,7^{\circ} \mathrm{C}$. Das Fieber wurde durch eine am folgenden Tage erneute Injection abermals hervorgerufen. Der folgende Tag war fieberfrei und zum Vergleiche geeignet. In dem zweiten Falle (Versuch 1) erschien der Patient symptomatisch beeinflusst (Kopfschmerz), eine Temperatursteigerung ist hier aber nicht erzielt worden. Die bei beiden Patienten vor dem Versuche pro Tag im Harn ausgeschiedenen Stickstoffmengen sind für jedes einzelne Individuum annähernd ubereinstimmend.

\section{Versuch 1 .}

Die betreffende Patientin ist eine $18 \mathrm{Jahr}$ alte Dienstmagd, welche seit drei Monaten an Symptomen der bacillären Lungenphthise leidet. Sie ist gegenwärtig fieberfrei und ohne Nachtschweisse. Links bestèt eine Dämpfung der Lungenspitze, an beiden Apices umschriebene Rasselgeräusche. Sie ist gross, schlank, hat ein Körpergewicht von $49,2 \mathrm{~kg}$.

*) Es ist das ältere Tuberculin Koch's gemeint. 
Ueber den Kohlenstoffgehalt des Harnes fiebernder Menschen u. s. w. 333

Mässig anämisch. In dem reichlichen schleimig-eiterigen Auswurf sind Tuberkelbacillen nicht nachweisbar.

Die Nahrungszufuhr während der Gesammtdauer des Versuches bestand aus:

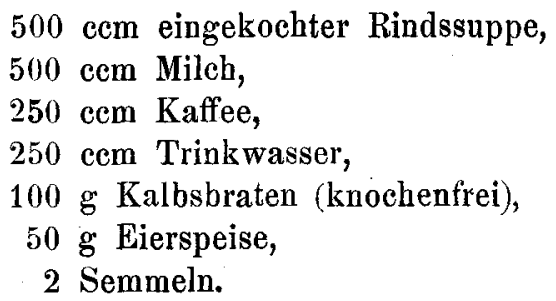

Am vierten und fünften Tage erhielt sie noch $1 / 8$ Liter Wein.

Am vierten Versuchstage wurden der Patientin um $8 \mathrm{~h}$ frib $0,01 \mathrm{~g}$ Tuberculinum Kochii injicirt. Nach der Injection beklagte sie sich um die Mittagszeit uber eingenommenen Kopf, doch blieb die Temperatur normal. Am fünften Tage erhielt das Versuchsindividunm um $8 \mathrm{~h}$ früh eine neuerliche Injection von $0,025 \mathrm{~g}$ Tuberculin. Um 2 h Nachmittags empfand sie Kopfschmerzen, subjectives Hitzegefiuhl, jedoch sorgfältige, einstündige Messung ergab keine Temperatursteigerung. Nachdem kein nachweisbares Fieber eintrat, wurde die Harnanalyse an diesem Tage unterlassen und der Versuch abgebrochen.

Tabelle III ertheilt Aufsehluss über die Resultate dieses Versuches.

Tabelue III.

\begin{tabular}{|c|c|c|c|c|c|c|c|c|c|c|c|c|}
\hline 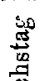 & $\boldsymbol{E}$ & & 总 & क्ष & 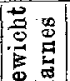 & In 5 & & & & In der & & \\
\hline & & & 4 & 胥. & 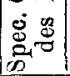 & $\left|\begin{array}{c}\mathrm{CO}_{2} \text { in } \\
\mathrm{g}\end{array}\right|$ & $\mathrm{C}$ in & $\begin{array}{c}N \text { in } \\
g\end{array}$ & & $\begin{array}{c}\mathrm{CO}_{2} \text { in } \\
\mathrm{g}\end{array}$ & $\begin{array}{c}\mathrm{C} \text { in } \\
\mathrm{g}\end{array}$ & $\begin{array}{c}N \text { in } \\
g\end{array}$ \\
\hline 1. & .7 & 6,8 & 49,2 & 900 & 1021 & 0,1481 & 404 & & 0,679 & & & \\
\hline 2. & $30 . / 7$. & $36,3-36,7$ & 49,3 & 850 & 1023 & 0,1698 &, 0463 & 30,0689 & $\{0,672$ & 28, & & 215 \\
\hline 3. & $31 . / 7$. & $36,4-36,8$ & 49,5 & 850 & 1025 & 0,1653 & 0,0451 & 1. 0,0684 & 0,659 & 12 & & 11,6323 \\
\hline 4. & $1 . / 8$. & $36,0-36,5$ & 404 & 1000 & 1020 & 0,1536 & 0,0419 & \begin{tabular}{l|l|l|}
9 & 0,0611
\end{tabular} & 0,686 & 30,7117 & 8,3759 & 12,2150 \\
\hline & 18 & $-37,1$ & 49,8 & 850 & 1022 & 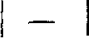 & 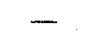 & 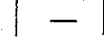 & - & & - & 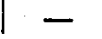 \\
\hline
\end{tabular}

Trotz Tuberculininjection blieb in vorliegendem Falle die Temperatur normal. Das Körpergewicht nahm eher etwas zu. Jedenfalls aber befand sich die Patientin in einem Vergiftungszustande, der in mancher Richtung einem infectiösen vergleichbar sein wird. Die geringe Harnmenge ist auf Transpiration infolge der heissen Jahreszeit und die ununterbrochene Bettruhe zuruckzuführen. Sowohl die 
Kohlenstoff- als auch die Stickstoffexcretion im Harne der ersten drei Versuchstage bewegt sich in normalen Grenzen und bleibt relativ sehr constant, ebenso wie das Verhältniss beider. Nach der Injection steigt die Ausfubr von Kohlenstoff und Stickstoff gegen den Vortag ein wenig, und zwar der Stickstoff um $0,5827 \mathrm{~g}$, also 4,8 Proc., der Kohlenstoff um 0,7119 g, entsprechend 8,5 Proc. Auch das Verhältniss beider erfährt eine Aenderung. Der Harn wird kohlenstoffreicher, und der Quotient $\frac{\mathrm{C}}{\mathrm{N}}$ erhebt sich gegen den Vortag um $0,027 \mathrm{~g}$, also 3,9 Proc.

\section{Versuch 2.}

Es handelt sich hier um einen 29 Jahre alten Müller, bei welchem sich seit einem Jahre die Symptome einer beiderseitigen bacillären Oberlappeninfiltration entwickelt haben. Ausserdem hatte er Lymphdrüsentuberculose und linksseitige Pleuritis sicca. Er war stark abgemagert und litt an Nachtschweissen. Das fruiher vorhandene Fieber hat seit mehreren Wochen aufgehört. Die eitrigen Sputa enthalten Tuberkelbacillen. Täglich ein fester Stuhl. Harn eiweissfrei.

Die Nahrung des Patienten bestand aus:

$500 \mathrm{ccm}$ eingekochter Suppe,

$500 \mathrm{ecm}$ Mileh,

$250 \mathrm{ccm}$ Wein,

$160 \mathrm{ccm}$ Wasser,

$100 \mathrm{~g}$ Kalbsbraten (knochenfrei),

$500 \mathrm{~g}$ Milchspeise,

2 Eiern,

2 Semmeln.

Am dritten und vierten Tage wurden $750 \mathrm{ccm}$ und am fünften Tage $500 \mathrm{cem}$ Trinkwasser verabfolgt, sonst blieb die Zufuhr die gleiche. Am zweiten Tage erbrach der Patient geringe Mengen gegen Abend und klagte über starke Brustschmerzen. Am dritten Tage erhielt er um $8 \mathrm{~h}$ früh eine subcutaue Injection von $5 \mathrm{mg}$ Tuberculin. Er hatte den ganzen Tag über keine subjectiven Beschwerden. Bis 2 h Mittag blieb die Temperatur innerhalb normaler Grenzen. Der Harn wurde bis zu dieser Zeit gesondert aufgefangen und analysirt. Um $3 \mathrm{~h}$ erhob sich die Temperatur, erreichte um $4 \mathrm{~h} 38,7^{\circ} \mathrm{C}$. und fiel dann langsam zur Norm ab. Der Harn von $2 \mathrm{~h}$ Nachmittags bis $8 \mathrm{~h}$ früh wurde als Fieberharn gesondert der Analyse unterworfen. Am vierten Tage versuchte ich, durch Injection einer grösseren Quantität ein höheres Fieber hervorzurufen. Der Patient erhielt um $8 \mathrm{~h}$ früh $10 \mathrm{mg}$ Tuberculin subcutan injicirt. Leider stieg die Temperatur nur bis $38,4^{0}$ C. ( $2 \mathrm{~h}$. Nachmittags). Der Harn wurde wie am 
Ueber den Kohlenstoffgehalt des Harnes fiebernder Menschen u. s. w. 335

Vortage wieder in zwei Portionen gesammelt und analysirt. Der Patient befand sich wohler als frïher. Das Stechen auf der Brust hatte nachgelassen. Der fünfte Tag, an welchem sich der Patient subjectiv sehr wohl befand, zeigte normale Temperaturen.

Tabelle IV und V geben eine Uebersicht der zur Beurtheilung des Versuches nöthigen Zahlen.

TABelle IV.

\begin{tabular}{|c|c|c|c|c|c|c|c|c|c|c|c|c|c|c|c|c|c|c|}
\hline $\begin{array}{l}\text { 罟 } \\
\text { 营 }\end{array}$ & 8 & 10 & 12 & 1 & 2 & 3 & 4 & 5 & 6 & 7 & 8 & 9 & 10 & 11 & 12 & 2 & 4 & 6 \\
\hline $4 . / 8$. & 36,1 & $36,0 \mid$ & 36,2 & - & 36,1 & - & 36,4 & - & 36,5 & - & 36,7 & - & 36,4 & - & 36,2 & 36,0 & 36,1 & 36,0 \\
\hline $5 . / 8$. & 36,0 & 36,1 & 36,0 & - & 36,2 & - & 36,4 & - & 36,5 & - & 36,3 & 一 & 36,1 & - & 36,2 & 36,4 & 36,0 & 36,2 \\
\hline $6 . / 8$. & 36,0 & 36,2 & 36,4 & 36,8 & 37,0 & 38,1 & 38,7 & 38,6 & 38,0 & 37,9 & 37,9 & 37,8 & 37,7 & 37,7 & 37,8 & 37,0 & 36,8 & 36,9 \\
\hline $7 . / 8$. & 36,3 & 36,4 & 37,9 & 38,0 & 38,4 & 38,3 & 38,3 & 38,2 & 38,4 & 38,3 & 38,3 & 38,1 & 38,2 & 37,9 & 37,5 & 37,2 & 36,9 & 36,8 \\
\hline $8 . / 8$. & 36,4 & 36,7 & $\overline{36}, 9$ & - & 37,0 & - & 37,4 & - & 37,0 & - & 36,9 & - & 36,2 & - & 36,1 & 36,5 & 36,4 & 36,1 \\
\hline
\end{tabular}

Tabelle V.

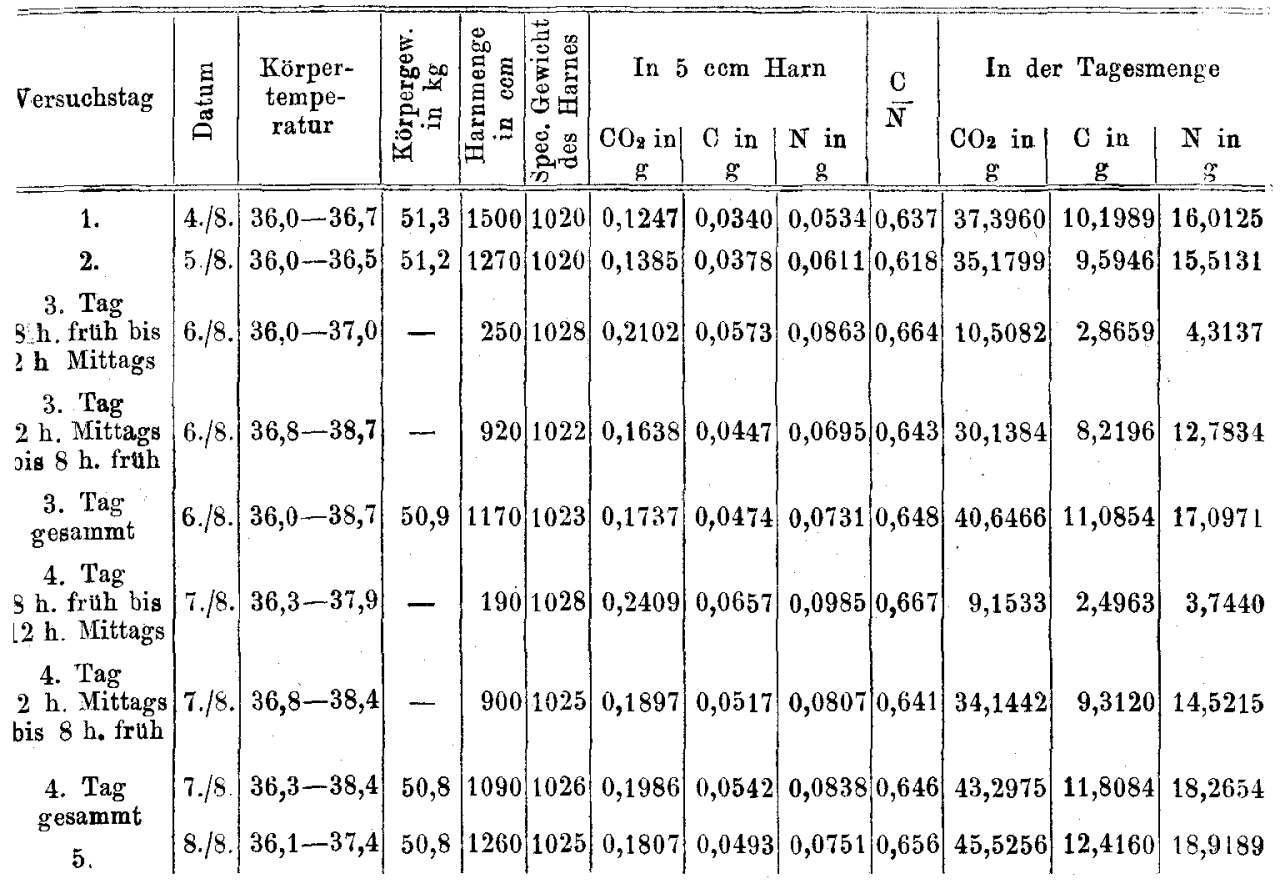


Bei diesem unzweifelhaft tuberculösen Kranken mit vorher normaler Temperatur gelang es, durch Tuberculininjectionen kurzwährendes Fieber (bis $38,7^{\circ}$ C.) zu erzeugen. Das Körpergewicht nahm während der Versuchszeit stetig ab ( $\mathrm{um} 0,5 \mathrm{~kg}$ ). Die Harnmenge war etwas gering, aber das specifische Gewicht ein entsprechend böheres. An den beiden dem Fieberversuch vorangehenden Tagen ist die Stickstoffausscheidung in 24 Stunden annähernd die gleiche. Die Kohlenstoffexcretion verhält sich ähnlich, auch der Quotient $\frac{\mathrm{C}}{\mathrm{N}}$ ist ziemlich constant. An den Tagen mit erhöhten Temperaturen war sowohl die Kohlenstoff- als die Stickstoffausscheidung durch den Harn eine grössere.

Vergleicht man den dritten (Fieber) Tag mit dem zweiten Versuchstag, an welchem normale Temperatur herrschte, so steigt die Kohlenstoffausscheidung während des Fiebers um 1,4908 g C., gleich 13,4 Proc., und die Stickstoffexcretion um 1,5840 g N, gleich 9,2 Proc. Der Quotient $\frac{\mathrm{C}}{\mathrm{N}}$ erbebt sich um $0,03=4,6$ Proc.

Aehnlich verhält sich der zweite Fiebertag. Vergleiche ich die Ausscheidung hier wiederum mit dem zweiten Versuchstage, so steigt die Kohlenstoffexcretion um 2,2138 g, gleich 18,7 Proc., und die Stickstoffexcretion um 2,7523 g; gleich 15 Proc. Auch in diesem Falle ist der Harn relativ etwas kohlenstoffreicher. Die Verhältnisszahl wird um $0,028=4,3$ Proc. grösser. Auffallend erscheint das Ergebniss des fünften (fieberfreien) Tages. Die Zahlen desselben sind die grössten der ganzen Versuchszeit. Vergleiche ich dieselben ubereinstimmend wie bei den Fiebertagen mit denjenigen des zweiten Tages, so finde ich ein Plus von 2,8214 g Kohlenstoff $=22,7$ Proc. und $3,4058 \mathrm{~g}$ Stickstoff $=18$ Proc. an diesem Tage. Auch der Quotient $\frac{\mathrm{C}}{\mathrm{N}}$ stieg um $0,038=5,8$ Proc.

Auf den Umstand, dass während eines einzelnen Versuchstages die in der fieberfreien Zeit und die während der Temperatursteigerung gesammelte Harnportion verschiedene Mengen Kohlenstoff enthalten, und infolge dessen der Quotient $\frac{\mathrm{C}}{\mathrm{N}}$ etwas verschieden ausfällt, lege ich natürlich kein grosses Gewicht, weil es sich um verschiedene Tagesperioden handelt.

Aus den beiden vorstehenden Versuchen könnte man in der That geneigt sein, auf eine wenn auch nur recht geringfügige stärkere Erhöhung der Kohlenstoffausscheidung gegenüber der Stickstoff- 
excretion im Sinne von May zu schliessen. Die Beobachtung (Versuch 2) einer noch stärkeren analogen Aenderung für den dem Fieber folgenden ohne Temperatursteigerung verlaufenden Tag könnte als Nachwirkung der Tuberculininjection gelten. Aehnliches beobachtete man ja auch früher, wenigstens hinsichtlich der Stickstoffausscheidung.

Hinsichtlich der Stickstoffexcretion im Tuberculinfieber ergeben meine Beobachtungen eine Bestätigung von frïheren ähnlichen Versuchen, welche von F. Hirsehfeld $d^{8}$, A. Loew $y^{9}$ ) und G. Klem perer10) ausgefuhrt worden sind. Auch in soweit muss ich Klemperer zustimmen, dass die Steigerung der Stickstoffausscheidung: nicht parallel mit der Temperatur läuft. Wenn Klemperer findet, dass im Verlaufe der Tuberculinbehandlung diese Steigerung völlig verschwinden kann, so sprechen meine Beobachtungen für die Möglichkeit einer Nachwirkung. Auch die Beobachtung von Loewy, dass bei ausbleibender Temperatursteigerung nach Injection von Tuberculin anch der Eiweisszerfall nicht beeinflusst wird, kann ich bestätigen.

\section{II.}

Daran reihe ich zwei weitere mit Malariakranken, welche natïrlich nicht mit Chinin behandelt wurden, angestellte Versuche. Diese, ebenso wie die mit Tuberculin behandelten, stehen der May'schen Versuchsanordnung insofern nahe, als das Fieber jedesmal relativ kurze Dauer besass. Nur der Uebelstand kommt bei den Malariakranken in Betracht, dass auch im günstigsten Falle Fieber und fieberfreie Zeit einander zu rasch folgen, so dass eventuelle Nachwirkungen störend sich geltend machen. Auch ist der Umstand, dass die Anfälle anteponirten, Schuld daran, dass in einem der beiden Versuche nicht volle 24 Stunden Normaltemperatur zur Beobachtung kam. Der andere Versuch bot einen völlig und einen fast fieberfreien Tag zum Vergleich. Wenn auch die pathologischen Temperatursteigerungen bei meinen Fällen nicht in völlig regelmässiger Folge mit normalen Perioden abwechseln, so ist doch, wenigstens in dem einen Falle (Versuch 3) der Vergleich gut möglich gewesen. Die Stickstoffausscheidung im Harne bei dem letzterwähnten Patienten war eine sehr gut vergleichbare, beziehungsweise relativ constante. Dagegen war bei dem Kranken in Versuch 4 eine stärkere Schwankung des Stickstoffgehaltes an den verschiedenen Versuchstagen zu constatiren. In beiden Fällen fanden sich Temperatursteigerungen bis über $40^{\circ} \mathrm{C}$.

$$
\text { Versuch } 3 \text {. }
$$

Der Patient war ein 48 jähriger Feldarbeiter, der seit Anfang Mai d. J. an Malaria gelitten hat. Gegenwärtig ist der Kranke auf ein Körper- 
gewicht von $52,9 \mathrm{~kg}$ abgemagert. Er hat jetzt Tertianfieber, wobei die Anfälle um etwa 3 Stunden zu anteponiren pflegen. Nach jedem Anfall starker Schweissausbruch. Mässig grosser, tastbarer Milztumor. Die Harnmenge wechselnd. Albuminurie besteht nicht. Im Blute spärliche Malariaplasmodien. Zahl der Erythrocyten 5200000 , der Leukocyten 4300.

Die Nahrung des Patienten bestand aus:

500 cem Milch,

1 Portion Obst (gedörrte Pflaumen),

$2000 \mathrm{ccm}$ Trinkwasser.

Vom zweiten Tage an erhielt er noch hinzu:

$500 \mathrm{ccm}$ Rindssuppe.

Am dritten Versuchstage sammelte ich den Harn in zwei Portionen, und zwar wurde in der ersten der Harn der fieberfreien Zeit von $8 \mathrm{~h}$ frulh bis $4 \mathrm{~h}$ Nachmittags, in der zweiten der Harn von $4 \mathrm{~h}$ Nachmittags bis $8 \mathrm{~h}$ frïh, in welcher Zeit das Fieber fiel, aufgefangen. In beiden Portionen wurden die entsprechenden Analysen ausgefilhrt und hierauf durch Rechnung die Werthe fur den Tagesharn bestimmt.

Die Temperatur, sowie die Ausscheidungsverhältnisse durch den Harn sind in den Tabellen VI und VII ersichtlich gemacht.

Tabelle VI.

\begin{tabular}{c|c|c|c|c|c|c|c|c|c|c|c|c|c|c}
\hline $\begin{array}{c}\text { Versuchs- } \\
\text { tag }\end{array}$ & Datum & 8 & 10 & 12 & 2 & 4 & 6 & 8 & 10 & 12 & 2 & 4 & 6 \\
\hline $\mathbf{1 .}$ & $2 . / 6$. & 36,2 & 39,9 & 39,1 & 38,9 & 38,6 & 37,0 & 36,2 & 36,1 & 36,0 & 36,1 & 36,0 & 36,0 \\
2. & $3 . / 6$. & 36,0 & 36,1 & 36,2 & 36,0 & 36,0 & 36,2 & 36,3 & 36,2 & 36,0 & 36,1 & 36,3 & 38,6 \\
3. & $4 . / 6$. & 39,1 & 39,1 & 38,6 & 37,8 & 37,7 & 36,5 & 36,9 & 36,5 & 36,2 & 36,4 & 36,2 & 36,0 \\
$\mathbf{4 .}$ & $5 . / 6$. & 36,0 & 36,0 & 36,2 & 36,1 & 36,0 & 36,2 & 36,5 & 36,2 & 36,8 & 36,4 & 38,5 & 40,3
\end{tabular}

TABelle VII.

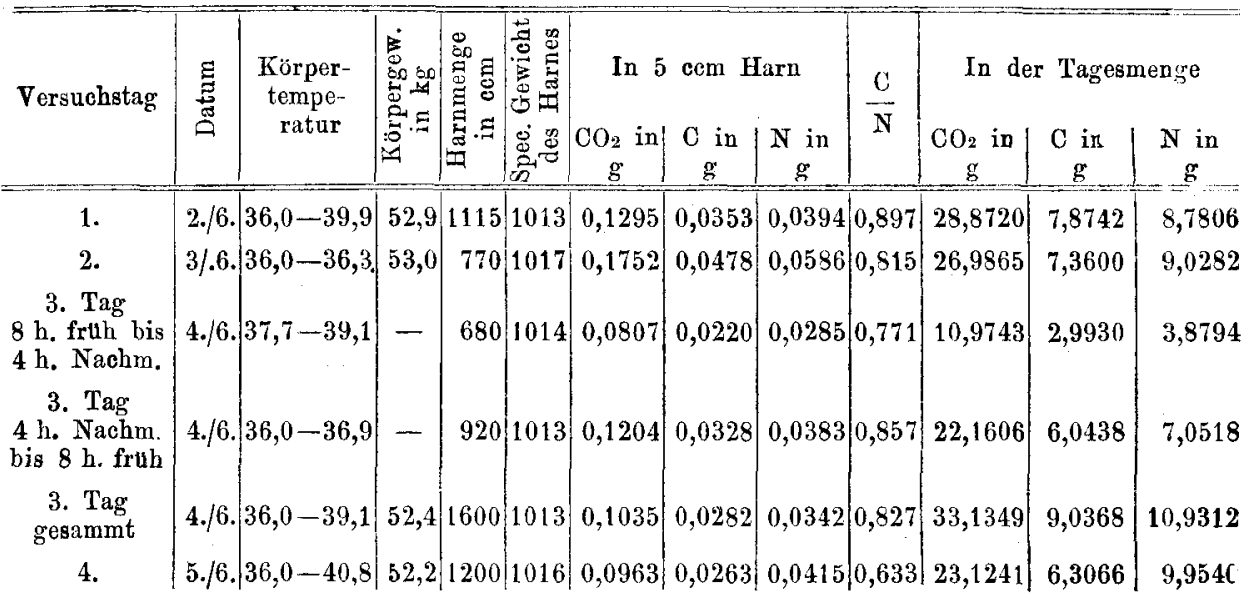


Das Körpergewicht des Patienten sinkt während der Versuchs. periode innerhalb vier Tagen um $0,7 \mathrm{~kg}$. Die Harnmenge zeigte nicht striet an den Fieberverlauf geknüpfte Schwankungen. Am ersten Versuchstage trat um $8 \mathrm{~h}$ früh bei $36,2^{\circ} \mathrm{C}$. Körpertemperatur Frostgefuhl ein, die Temperatur erhob sich rasch auf $39,9^{\circ} \mathrm{C} .(10 \mathrm{~h}$ Vormittags), sank hierauf allmählich, und um 4 h Nachmittags erfolgte Schweissausbruch. Bereits um $5 \mathrm{~h}$ Nachmittags war die Temperatur normal und blieb in diesen Grenzen auch am zweiten Versuchstage. Jedoch schon um $6 \mathrm{~h}$ friih trat wieder Frostgefühl auf. Der Patient entleerte hierauf seine Blase, so dass der zweite Tag als Normaltag zu betrachten ist. Um $8 \mathrm{~h}$ früh des dritten Versuchstages hatte die Temperatur bereits $39,1^{\circ} \mathrm{C}$. erreicht. Der Harn wurde nun bis $4 \mathrm{~b}$ Nachmittags gesondert aufgefangen, zu welcher Zeit nach vorausgegangenem Schweissausbruch die Temperatur wieder normal geworden war. Patient musste um $4 \mathrm{~h}$ seine Blase entleeren, und dieser Harn wurde noch der ersten Portion hinzugegeben. Die zweite Portion umfasst den Harn einer völlig fieberfreien Zeit. Leider anteponirte das Fieber um eirca 4 Stunden, so dass der vierte Tag zwar kein völlig, aber grösstentheils fieberfreier war. Um $3 \mathrm{~h}$ Morgens trat dann abermals Frost und hierauf Anstieg der Temperatur ein. Der Vergleich der Resultate des ersten (Fieber) Tages und des zweiten (fieberfreien) Tages zeigte eine deutliche Mehrausscheidung von Kohlenstoff, sowie eine (um 9 Proc.) grössere Verhältnisszahl $\frac{C}{N}$ am ersten Tage, während der Stickstoffgehalt des Fieberharnes etwas geringer ist. Der dritte Tag (Fieber) in seiner Gesammtheit ergiebt ebenfalls eine Steigerung der Kohlenstoff- und Stickstoffexcretion and eine geringe Erhöhung der Verbältnisszahl beider im Vergleiche zu dem zweiten fieberfreien Tage. Die Resultate der Analyse beider Portionen, sowohl des Fieber- als Normalharnes dieses Tages sind nicht ohne weiteres, wie schon erwähnt, mit einander vergleichbar, nachdem die erste Partie 8, die zweite 16 Stunden umfasst und beide Portionen anderen Tageszeiten angehören, in welchen die Stoffzufuhr eine verschiedene war. Der Harn der fieberfreien Zeit hat einen grösseren Quotienten $\frac{\mathrm{C}}{\mathrm{N}}$ als der Fieberharn. In den vierten Tag, der, wie schon erwähnt, grösstentheils ohne Temperatursteigerung verlief, fällt gegen Morgen der Beginn eines Fieberanfalles, weshalb diese Versuchszahlen nicht vollkommen als Normale verwerthet werden könnien. Auffallend ist der geringe Werth des Verbältnisses $\frac{C}{N}$ 
$(0,633)$ an diesem Tage gegenüber Werthen der vorhergehenden Tage (erster Tag: 0,897, daher ein Untersebied von 0,264, gleich 29,4 Proc.). Bei der Möglichkeit, dass wegen der fortwährend einander folgenden Fieberanfälle sich Nachwirkungen geltend machen, kann dieses Verhalten weder für, noch gegen die May'sche Anschaung verwerthet werden. Der Vergleich des ersten Fiebertages und des zweiten ohne Fieber verlaufenen Tages würde eventuell für die Annahme May's sprechen. -

\section{Versuch 4.}

18 Jahre alter Rumäne, welcher schon seit dem 13. Jahre an Intervallen von Malaria leidet. Vor Kurzem neuerdings Attaque von Febris tertiana. Graciler Mensch von $61 \mathrm{~kg}$ Körpergewicht. Anämisches Anssehen. In den Anfällen, welche bis 10 Stunden dauerr, werden Temperaturen bis tiber $40^{\circ} \mathrm{C}$. beobachtet; nach deren Abfall starker Schweiss. In den fieberfreien Intervallen Woblbefinden. Typischer Milztumor, welcher den Rippenbogen um zwei Querfinger iberschreitet. Im Blut der Parasit nachweisbar. Keine Albuminurie.

Die Nahrungszufuhr des Patienten besteht aus:

$$
\begin{aligned}
& 750 \text { cem Milch, } \\
& 600 \text { cem Suppe, } \\
& 300 \text { cem Wein, } \\
& 1250 \text { cem Wasser, } \\
& 4 \text { Broten }(200 \mathrm{~g}) .
\end{aligned}
$$

Eine völlig gleichförmige Ernährung war leider nicht durchzuführen. Bereits am vierten Tage (zweiter Versuchstag) erbrach der Kranke um $1 / 26 \mathrm{~h}$ fruih heftig und verlangte dringend eine andere Nahrung. Es wurde ihm deshalb zugestanden:

$$
\begin{array}{rl}
500 & \mathrm{ccm} \text { Milch, } \\
300 \mathrm{ccm} \text { Suppe, } & \mathrm{ccm} \text { Wein, } \\
500 & \mathrm{ccm} \text { Wasser, } \\
1000 & \mathrm{~g} \text { Schinken, } \\
60 & \mathrm{~g} \text { Eierspeise, } \\
4 & \text { Brode }(200 \mathrm{~g}) .
\end{array}
$$

Am dritten, sowie am vierten Tage erhielt er nur $250 \mathrm{cem}$ Milch, dafür $250 \mathrm{ccm}$ Thee und $250 \mathrm{ccm}$ Wein; sonst die gleiche Zufuhr, wie am Vortage. Am vierten Tage wurde der Harn in zwei Portionen gesammelt und nur der Fieberharn der Analyse unterworfen.

Tabelle VIII und IX geben ein Bild der Temperaturschwankungen und der Excretionsverhältnisse durch den Harn. 
Ueber den Kohlenstoffgehalt des Harnes fiebernder Menschen n. s. w. 341

TABeLLE VIII.

\begin{tabular}{c|c|c|c|c|c|c|c|c|c|c|c|c|c}
\hline $\begin{array}{c}\text { Versuchs- } \\
\text { tag }\end{array}$ & Datum & 8 & 10 & 12 & 2 & 4 & 6 & 8 & 10 & 12 & 2 & 4 & 6 \\
\hline 1. & $17 . / 6$. & 38,2 & 39,8 & 40,4 & 39,6 & 38,3 & 37,8 & 37,0 & 36,8 & 36,7 & 36,5 & 36,2 & 36,2 \\
2. & $18 . / 6$. & 36,0 & 36,0 & 36,2 & 36,4 & 36,9 & 37,3 & 37,5 & 38,3 & 38,8 & 39,0 & 39,4 & 40,1 \\
3. & 19.6. & 39,7 & 39,2 & 38,9 & 37,3 & 37,0 & 37,0 & 36,8 & 36,7 & 36,7 & 36,5 & 36,2 & 36,2 \\
4. & $20 . / 6$. & 36,1 & 36,2 & 36,4 & 37,3 & 38,4 & 38,6 & 38,9 & 39,3 & 38,5 & 38,5 & 38,3 & 38,3
\end{tabular}

TABELLE IX.

\begin{tabular}{|c|c|c|c|c|c|c|c|c|c|c|c|c|}
\hline \multirow{2}{*}{$\begin{array}{c}\text { Versuchs- } \\
\text { tag }\end{array}$} & \multirow{2}{*}{ 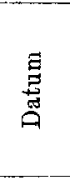 } & \multirow{2}{*}{$\begin{array}{c}\text { Körper- } \\
\text { tempe- } \\
\text { ratur }\end{array}$} & \multirow{2}{*}{ 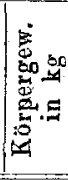 } & \multirow{2}{*}{ 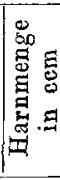 } & \multirow{2}{*}{ 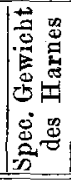 } & \multirow{2}{*}{$\begin{array}{c}\text { In } 5 \\
\mathrm{CO}_{2} \text { in } \\
\mathrm{g}\end{array}$} & $\mathrm{ccm}$ & Harn & \multirow{2}{*}{$\frac{\mathrm{C}}{\mathrm{N}}$} & \multicolumn{3}{|c|}{ In der Tagesmenge } \\
\hline & & & & & & & $\begin{array}{c}\mathrm{C} \text { in } \\
\mathrm{g}\end{array}$ & $\begin{array}{c}N \text { in } \\
g\end{array}$ & & $\begin{array}{c}\mathrm{CO}_{2} \text { in } \\
\mathrm{g}\end{array}$ & $\mathrm{C}$ in & $\begin{array}{c}N \text { in } \\
g\end{array}$ \\
\hline 1. & $17 . / 6$. & $36,2-40,4$ & 61,0 & 700 & 1021 & 0,2445 & 0,0667 & 0,0880 & 0,757 & 34,2285 & 9,3350 & 12,3235 \\
\hline 2. & 18./6. & $36,0-40,1$ & $61,0\}$ & 1140 & 1026 & 0,2394 & 0,0653 & 0,0906 & $0,720\}$ & 54,5765 & 14,8845 & 20,6682 \\
\hline 3. & 19./6. & $36,2-39,7$ & 62,0 & 530 & 1020 & 0,2168 & 0,0591 & 0,0751 & 0,787 & 22,9796 & 6,2671 & 7,9579 \\
\hline $\begin{array}{l}4 . \\
\text { h. fruh bis } \\
\text { h. Nachm. }\end{array}$ & $20 . / 6$. & $36,1-37,3$ & 61,9 & 640 & 1025 & 0,2529 & 0,0690 & 0,0940 & 0,734 & 32,3737 & 8,8292 & 12,0288 \\
\hline
\end{tabular}

Leider war auch in diesem Falle das Fieber ein anteponirendes. Es erschien bei jedem Anfalle um fast 5 Stunden früher, so dass auf jeden Tag erhöhte Temperaturen fielen. Das Sammeln des 24stündigen Harnes auf eine andere Zeit als von $8 \mathrm{~h}$ früh bis zur gleichen Stunde des folgenden Tages zu verlegen, erschien ebenfalls bei diesem Versuchsindividuum nicht möglich, da auch unter dieser Versuchsanordnung keine 24 stilndige fieberfreie Periode hätte gewonnen werden können. Nachdem es weiterhin aus äusseren Gründen unmöglich war, den Patienten ohne therapeutischen Eingriff zu belassen, wurde der Harn am vierten Tage von $8 \mathrm{~h}$ früh bis $4 \mathrm{~h}$ Nachmittags nach vollständiger Entleerung der Blase gesammelt und diese Harnportion der Analyse unterworfen. Während dieser Zeit bestand normale Temperatur. Der Patient erbielt nun $1 \mathrm{~g}$ Chinin. Von $4 \mathrm{~h}$ Nachmittags bis $8 \mathrm{~h}$ frib wurde eine Menge von $480 \mathrm{ecm}$ Harn mit dem specifischen Gewicht von 1023 aufgefangen. In demselben war Chinin nachweisbar. Im Verlaufe der letzten drei Versuchstage hatte Patient keinen Stuhl abgesetzt. Während des Versuches nahm bei relativ reichlicher Ernährung das Körpergewicht des Patienten (um fast $1 \mathbf{k g}$ ) zu. Die Harnmenge war eine wechselnde, ebenso das specifische Gewicht. Der Kohlenstoff- und Stickstoffgehalt des Harnes war schwankend mit der Menge des letzteren, so dass die Excretion in 
je $5 \mathrm{ccm}$ eine ziemlich gleichförmige war. Das Verhältniss $\mathrm{C}: \mathrm{N}$ zeigte sich in der Harnportion der fieberfreien Zeit des vierten Tages kleiner als in der 24 stündigen Menge der vorhergehenden Tage. Weitergehende Schliusse wären aus diesen Resultaten allerdings kaum einwandsfrei.

\section{III.}

Schliesslich berichte ich noch aber zwei Versuche, deren erster an einem von Angina befallenen Individuum, deren zweiter bei einem pneumonischen Patienten in der Nähe der Krise angestellt worden ist, und endlich noch über zwei weitere an mit Typhus unternommenen. Ich vereinige diese Versuche in eine Gruppe, weil es sich in denselben nicht um kurzdauernde Fieberanfälle, sondern um etwas länger währende natürliche Infecte handelt. In allen vier Fällen kam die fieberhafte Zeit und die folgende Periode der Entfieberung in Vergleich. Nur einen der Versuche trifft der Vorwurf, dass die Bestimmungsgrössen gewonnen worden sind, während der Patient sich in einem wesentlich verschiedenen Zustand der Ernährung befand. Ich werde naturgemäss auf diesen letzteren Versuch auch nur geringeres Gewicht legen.

\section{Versuch 5 .}

Der 26 Jahre alte Patient, der schon öfter an Halsentzundung gelitten hat, erkrankte am 15. Mai 1897 nach einer Verkältung abermals an Angina. Er ist ein $51 \mathrm{~kg}$ schwerer Mann. Die Vergrösserung der Tonsillen ist eine bedeutende. Die Halslymphdrïsen sind geschwollen.

Die Nahrung des Patienten besteht während des Fiebers aus:

$$
\begin{aligned}
& 500 \text { ccm Wein, } \\
& 500 \text { ccm Milch, } \\
& 800 \text { ccm Trinkwasser, } \\
& 1 \text { Portion Milchspeise, } \\
& 1 \text { Kipfel. }
\end{aligned}
$$

Mit dem zweiten Versuchstage mussten dem Patienten täglich noch mehr verabfolgt werden:

$$
\begin{aligned}
& 1 \text { Portion Obst (gedörrte Pflaumen), } \\
& 150 \text { ccm Weinsuppe. }
\end{aligned}
$$

Im Harn leichte Albuminurie. Täglich ein fester Stuhl.

Die Tabellen X und XI bringen eine Uebersicht der Temperaturund Ausscheidungsverhältnisse durch den Harn während der Versuchszeit. 
Ueber den Kohlenstoffigehalt des Harnes fiebernder Menschen u. s. w.

TABELLE X.

\begin{tabular}{c|c|c|c|c|c|c|c|c|c|c|c|c|c}
\hline Versuchs- & Datum & 8 & 10 & 12 & 2 & 4 & 6 & 8 & 10 & 12 & 2 & 4 & 6 \\
\hline \hline 1. & $19 . / 5$. & 38,6 & 38,7 & 39,4 & 39,2 & 39,6 & 39,3 & 39,2 & 39,0 & 38,9 & 38,7 & 38,5 & 38,0 \\
$\mathbf{2 .}$ & $20 . / 5$. & 37,9 & 38,0 & 38,1 & 38,2 & 38,9 & 39,0 & 38,7 & 38,6 & 38,8 & 38,5 & 38,1 & 37,4 \\
3. & $21 . / 5$. & 37,4 & 37,2 & 37,1 & 37,2 & 37,3 & 37,9 & 38,2 & 38,0 & 37,9 & 37,4 & 37,0 & 36,8 \\
4. & $22 . / 5$. & 36,0 & 36,1 & 36,4 & 36,5 & 36,2 & 36,4 & 36,8 & 37,4 & 37,2 & 37,3 & 37,1 & 37,0
\end{tabular}

TABerle XI.

\begin{tabular}{|c|c|c|c|c|c|c|c|c|c|c|c|c|}
\hline 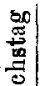 & छ & & 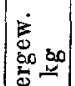 & 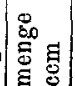 & 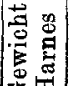 & In 5 & $5 \mathrm{ccm}$ & Harn & G & In de & Tagesn & menge \\
\hline 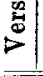 & 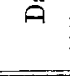 & & $=$ & 胥. & 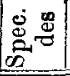 & $\begin{array}{c}\mathrm{CO}_{2} \text { in } \\
\mathrm{g}\end{array}$ & $\begin{array}{c}\mathrm{C} \text { in } \\
\mathrm{g}\end{array}$ & $\mid \begin{array}{c}\mathrm{N} \text { in } \\
\mathrm{g}\end{array}$ & IN & $\begin{array}{r}\mathrm{CO}_{2} \text { in } \\
\mathrm{g}\end{array}$ & $\begin{array}{c}\mathrm{C} \text { in } \\
\mathrm{g}\end{array}$ & $\begin{array}{c}N \text { in } \\
g\end{array}$ \\
\hline 1. & $19 . / 5$. & 38,0 & 1,0 & 730 & & & & & 0,174 & & & \\
\hline 2. & $20 . / 5$. & $37,4-39,0$ & 51,0 & 530 & 1021 & 0,2445 & 0,0667 & 70,0894 & 0,734 & 25,9158 & 7,0669 & 9,4790 \\
\hline 9 & $21 . / 5$. & $36,8-38,2$ & 50,5 & 1280 & 1019 & 0,2026 & 0,0553 & 30,0702 & 0,787 & 51,8751 & 14,1477 & 17,9648 \\
\hline & $-x$ & & a d & 100 & L & 0191 & 0299 & 3004 & $0=0$ & & & 73 \\
\hline
\end{tabular}

Das Fieber erreichte $39,6^{\circ} \mathrm{C}$. am ersten Tage, sank allmählich und am vierten Versuchstage trat normale Temperatur ein. Das Körpergewicht verringerte sich innerhalb dieser Zeit um 0,6 kg. Die Harnmenge war gering, besonders am zweiten Tage, trotz gesteigerter Zufuhr; stieg aber mit eintretender Entfieberung bedeutend, zugleich mit Herabminderung des specifischen Gewichtes. Die Ausscheidung: des Kohlenstoffes und Stickstoffes durch den Harn war während der Zeit hohen Fiebers eine geringere als am dritten Tage und bei der Entfieberung. Die Differenz der Kohlenstoffausfuhr am fieberfreien Tage gegenüber dem ersten Tag (Fieber) beträgt 1,1032 g $=7,8$ Proc. und die Differenz der Stickstoffexcretion 1,2868 $\mathrm{g}=7,1$ Proc. Der Quotient $\frac{\mathrm{C}}{\mathrm{N}}$ erlitt keine bedeutende Verschiebung, war aber am dritten Tage (höchste Temperatur $38,2^{\circ} \mathrm{C}$ ), sowie am Normaltage höher als in der Zeit hoher Temperaturen, er verhält sich also eher im Gegensatze zu der Aufstellung May's.

\section{Versuch 6.}

Der 12 Jahre alte Schüler J. G. bekam in der Nacht rom 16. zum 17. März Schïttelfrost und nachher Fieber. Seither entwickelte sich eine P n e u m on i e des rechten Unterlappens. Das Körpergewicht des Patienten betrug bei der Aufnahme 26,5 kg. Die Temperatur schwankte zwischen 38,6 bis $40,0^{0} \mathrm{C}$. 
Der Patient erhielt folgende Nahrung:

$$
\begin{aligned}
& 1000 \text { cem Milch, } \\
& 250 \text { ccm Rindssuppe mit Ei, } \\
& 150 \text { cem Weinsuppe, } \\
& 150 \text { ccm Wein, } \\
& 500 \text { cem Trinkwasser. }
\end{aligned}
$$

Infolge grossen Hungergefuhles mussten dem Patienten am letzten Versuchstage (23. März) noch bewilligt werden:

$60 \mathrm{~g}$ Schinken,

2 Semmeln,

$50 \mathrm{ccm}$ Wein.

Der Harn ist dunkelgefärbt, gering an Menge, eiweissfrei. Täglich ein fester Stuhl.

Die Temperaturschwankungen wäbrend der Versuchstage sind in Tabelle XII ersichtlich:

Tabelle XII.

\begin{tabular}{|c|c|c|c|c|c|c|c|c|c|c|c|c|}
\hline \multirow{2}{*}{ 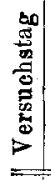 } & \multirow{2}{*}{ 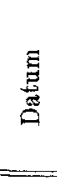 } & \multirow{2}{*}{$\begin{array}{c}\text { Körper- } \\
\text { tempe- } \\
\text { ratur }\end{array}$} & \multirow{2}{*}{ 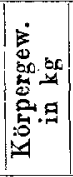 } & \multirow{2}{*}{ 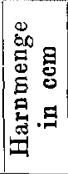 } & \multirow{2}{*}{ 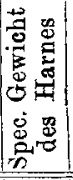 } & \multirow{2}{*}{$\begin{array}{c}\text { In } 5 \\
\mathrm{CO}_{2} \text { in } \\
\mathrm{g}\end{array}$} & \multirow{2}{*}{$\begin{array}{c}\mathrm{C} \text { in } \\
\mathrm{g}\end{array}$} & \multirow{2}{*}{$\begin{array}{l}\text { Harn } \\
\mid \begin{array}{c}\mathrm{N} \text { in } \\
\mathrm{g}\end{array}\end{array}$} & \multirow{2}{*}{$\frac{\mathrm{C}}{\mathrm{N}}$} & \multicolumn{3}{|c|}{ In der Tagesmenge } \\
\hline & & & & & & & & & & $\mathrm{g}$ & $\mathrm{C}$ in & \\
\hline 1. & & & 26,5 & & 1020 & & 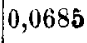 & 00914 & & & 0,0002 & \\
\hline 2. & & & 26,5 & 620 & 1020 & & & 0,0888 & $0,0,0$ & & 8,6333 & \\
\hline & $3 . / 3$ & 6,7 & $|26,4|$ & 760 & 1019 & $804 T_{1}$ & 0,053 & 0,0762 & ,699 & $|29,7024|$ & 8,1007 & |11,,5843 \\
\hline
\end{tabular}

\begin{tabular}{c|c|c|c|c|c|c|c|c|c|c|c|c|c}
\hline $\begin{array}{c}\text { Versuchs- } \\
\text { tag }\end{array}$ & Datum & 8 & 10 & 12 & 2 & 4 & 6 & 8 & 10 & 12 & 2 & 4 & 6 \\
\hline \hline 1. & $21 . / 3$. & 38,7 & 38,9 & 39,0 & 39,0 & 39,1 & 38,0 & 37,7 & 37,4 & 37,0 & 36,8 & 36,5 & 36,7 \\
2. & $22 . / 3$. & 36,0 & 36,5 & 37,0 & 37,0 & 36,6 & 36,5 & 36,6 & 36,3 & 36,0 & 36,2 & 36,4 & 36,1 \\
3. & $23 . / 3$. & 36,4 & 36,2 & 36,4 & 36,4 & 36,5 & 36,5 & 36,7 & 36,7 & 36,5 & 36,6 & 36,7 & 36,7
\end{tabular}

Tabelle XIII orientirt über die Ausscheidungsverhältnisse durch den Harn.

Tabelle XIII.

Bei diesem Versuche tritt bereits am ersten Versuchstage, dem 6. Fiebertage, die Krise ein. Die Temperatur sinkt rasch von $39,1^{\circ} \mathrm{C}$. zu normalen Werthen. Die vorher trotz bedeutender Fltissigkeitsaufnahme geringe Harnmenge hebt sich am zweiten Tage der Ent- 
fieberung unbedeutend, zugleich mit einer geringen Erniedrigung des specifischen Gewichtes. In der Ausscheidung von Kohlenstoff und Stickstoff durch den Harn erfolgt keine bedeutende Aenderung. An dem der Krise folgenden Tag steigt die Kohlenstoffexcretion etwas (um $0,2781 \mathrm{~g}=3,2$ Proc.), während der Stickstoffgehait des Harnes eher ein wenig sinkt (um $0,1427 \mathrm{~g}=1,3$ Proc.), so dass der Quotient $\frac{\mathrm{C}}{\mathrm{N}}$ grösser als am Fiebertage wird. Und am zweitfolgenden Tage mit normaler Temperatur erfolgt abermals eine Aenderung des Verhältnisses $\frac{\mathrm{C}}{\mathrm{N}}$, sodass der Quotient gegen den Fiebertag um etwa 6,6 Proc. herabgemindert wird. Die Aenderung des Quotienten $\frac{C}{N}$. bewegt sich also hier im Sinne von May. Dass aber wenigstens nicht etwa der Temperaturverlauf als solcher hier das Maassgebende ist, geht daraus hervor, dass an dem ersten der Krise folgenden Tag der Quotient wirklich höher ist als am Tage der Krise. Der Patient befindet sich während des ganzen Versuches annähernd im Stickstoffgleichgewicht. -

\section{Versuch 7 .}

33 jähriger Mann, der seit 2 Wochen an einem leichten Typhus abdominalis leidet. Zur Zeit des Beginnes des Versuches befindet er sich etwa in der dritten Krankheitswoche. Seit Anfang der Krankheit ist er bereits etwas abgemagert. Das Körpergewicht beträgt jetzt $52,9 \mathrm{~kg}$. Die Temperatur schwankt zwischen 38,5-400 C., die Pulsfrequenz übersteigt nicht 90 Schläge. Kein ausgeprägter Status typhosus. Mässig: grosse Milzgeschwulst. Zwei Stihle pro Tag. Hypoleukocytose. Positive Diazoreaction. Kein Eiweiss im Harne.

Patient erhält bereits seit drei Tagen und ebenso durch die Versuchszeit folgende Nahrung:

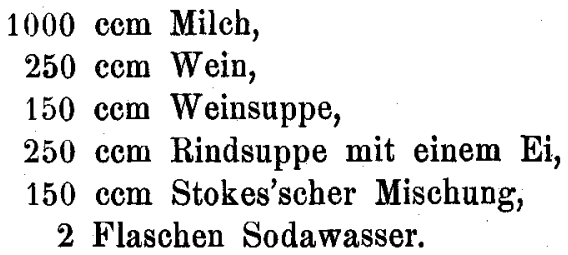

In den zwei letzten Versuchstagen erhielt Patient noch eine Portion Chaudeau, bestehend aus $1 / 10$ Liter Wein, 2 Eidotter und 1 Löffel Zucker.

Die Temperaturschwankungen sind aus Tabelle XIV za entnehmen. 
TABelle XIV.

\begin{tabular}{c|c|c|c|c|c|c|c|c|c|c|c|c|c}
\hline $\begin{array}{c}\text { Versuchs- } \\
\text { tag }\end{array}$ & Datum & 8 & 10 & 12 & 2 & 4 & 6 & 8 & 10 & 12 & 2 & 4 & 6 \\
\hline 1. & $11 . / 3$. & 38,4 & 38,6 & 38,8 & 39,1 & 39,4 & 39,5 & 39,6 & 39,5 & 39,3 & 39,0 & 38,7 & 38,5 \\
$\mathbf{2 .}$ & $12 . / 3$. & 38,0 & 37,9 & 37,6 & 37,7 & 38,9 & 38,9 & 38,6 & 38,1 & 37,9 & 37,6 & 37,3 & 37,4 \\
3. & $13 . / 3$. & 38,1 & 38,6 & 38,3 & 38,5 & 38,4 & 38,6 & 38,8 & 38,9 & 39,7 & 37,8 & 37,6 & 37,3 \\
$\mathbf{4 .}$ & $14 . / 3$. & 37,1 & 38,0 & 38,6 & 39,1 & 37,1 & 37,8 & 37,9 & 37,6 & 37,8 & 37,9 & 38,1 & 37,6 \\
$\mathbf{5 .}$ & $15 . / 3$. & 37,1 & 38,0 & 38,1 & 38,2 & 38,6 & 38,7 & 38,9 & 38,8 & 38,6 & 38,8 & 38,4 & 38,2 \\
6. & $16 . / 3$. & 37,0 & 37,2 & 37,3 & 38,5 & 38,7 & 38,6 & 38,2 & 38,0 & 37,9 & 38,0 & 37,8 & 37,7 \\
7. & $17 . / 3$. & 36,7 & 36,5 & 36,6 & 37,5 & 37,7 & 37,9 & 38,1 & 37,9 & 37,3 & 37,1 & 36,9 & 36,8 \\
8. & $18 . / 3$. & 36,5 & 36,4 & 36,3 & 36,2 & 37,4 & 37,3 & 37,4 & 37,3 & 37,1 & 37,2 & 36,9 & 36,4 \\
$\mathbf{9 .}$ & $19 / 3$. & 36,2 & 36,3 & 36,5 & 37,0 & 37,4 & 37,5 & 37,5 & 37,4 & 37,2 & 37,5 & 37,3 & 37,0
\end{tabular}

Die näcbstfolgende Tabelle bietet eine Uebersicht über die Ausscheidungsverhältnisse von Kohlenstoff und Stickstoff durch den Harn.

Tabelle XV.

\begin{tabular}{|c|c|c|c|c|c|c|c|c|c|c|c|}
\hline \multirow{2}{*}{ 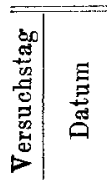 } & \multirow{2}{*}{$\begin{array}{l}\text { Körper- } \\
\text { tempe- } \\
\text { ratur }\end{array}$} & \multirow{2}{*}{\multicolumn{2}{|c|}{ 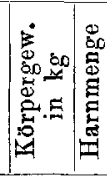 }} & \multirow{2}{*}{ 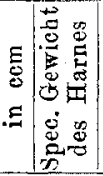 } & \multicolumn{2}{|r|}{$5 \mathrm{ccm}$} & Harn & \multirow{2}{*}{$\frac{\mathrm{C}}{\mathrm{N}}$} & \multicolumn{3}{|c|}{ Im Tagesharn } \\
\hline & & & & & $\underset{\mathrm{g}}{\mathrm{CO}_{2} \text { in }}$ & $\mathrm{C}$ in & $N_{g}$ in & & $\underset{g}{\mathrm{CO}_{2}}$ in & $\mathrm{C}$ in & $\mathrm{N}$ in \\
\hline
\end{tabular}

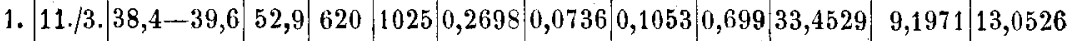

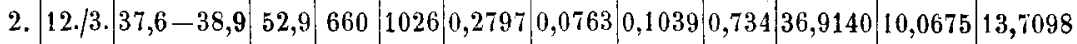

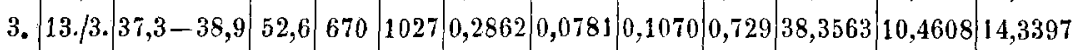

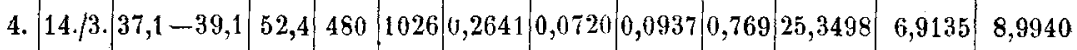

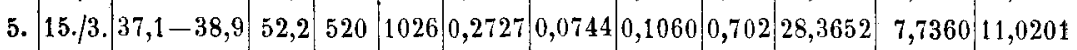

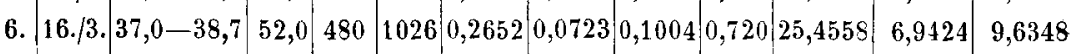

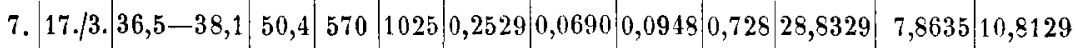

\begin{tabular}{l|l|l|l|l|l|l|l|l|l|l|l|l|l|l|l|l|l|l|l|} 
8. $18 . / 3$. & $36,2-37,4$ & 50,1 & 75 & 1016 & 0,1505 & 0,0411 & 0,0577 & 0,712 & 23,3349 & 6,3640 & 8,9377
\end{tabular}

9. $19 / 3.36,2-37,5|50,0| 990|1014| 0,1216|0,0332| 0,0458|0,725| 24,0852|6,5687| 9,0610$

Das Fieber des Patienten, welches am ersten Versucbstag $39,6^{\circ} \mathrm{C}$. erreicht hatte, fällt beständig, so dass am achten und neunten Versuchstag bereits normale Temperaturen beobachtet werden. Das Körpergewicht sinkt wäbrend dieser Zeit von 52,9 auf $50,0 \mathrm{~kg}$, also um fast $3 \mathrm{~kg}$. An den Fiebertagen ist der Harn spärlich und dunkel gefärbt. Mit Eintritt der Entfieberung nimmt die Harnausscheidung zu und das specifische Gewicht des Urins wird geringer. Der Patient scheidet vom vierten bis neunten Versuchstag ziemlich vergleichbare Stickstoffmengen aus. Während des Fiebers ist die Koblenstoff- und 
Stickstoffexcretion ersichtlich grösser als in der fieberfreien Zeit. Die Mehrausscheidung des Kohlenstoffes am dritten Versuchstag, an welchem die Excretion am grössten gefunden wurde, im Vergleich zum Mittel der zwei letzten fieberfreien Tage, beträgt etwa $4 \mathrm{~g}$, also ungefähr 40 Proc. Während sich hinsichtlich des Stickstoffes für dieselben Tage eine Differenz von $5,3 \mathrm{~g}$, gieich 37 Proc. ergeben hat. Eine Abweichung des Quotienten $\frac{\mathrm{C}}{\mathrm{N}}$ während der Fiebertage von derselben Relation nach der Entfieberung war nicht zu beobachten. Es findet sich nämlich der Quotient $\frac{\mathrm{C}}{\mathrm{N}}$ während der sieben Fiebertages stark schwankend. Werthen von 0,769 stehen solche von 0,699 gegenuiber (Differenz 9,1 Proc). Ein Mittel aus diesen Werthen wird kaum gezogen werden diurfen. Am dritten, fünften, sechsten und siebenten Versuchstag, also an vier Fiebertagen, ist der Werth identisch mit dem der Werthe nach der Entfieberung.

Versuch 8.

42 Jahre alter Gerber, der am 12. Mai 1897 die Prodrome des Typhus bekam. Der Beginn des Fiebers fällt vermuthlich etwa am 20. d.M. Im Anfange der Versuchsperiode beträgt das Körpergewicht $61,7 \mathrm{~kg}$. Das Sensorium ist relativ frei. Während der Versuchsperiode besteht ein mässig hohes Fieber. Roseola. Bronchitis. Pulsfrequenz 90 bis 96 . Palpable Milz. Leichte Albuminurie und Nukleoalbuminurie. Keine Diazoreaction. Starke Diarrhoe. Geringe Hypoleukocytose.

Die Nahrung besteht aus:

$600 \mathrm{ecm}$ Milch.

$1000 \mathrm{cem}$ Trinkwasser.

Es ist dies jener Versuch, der insofern nicht vollkommen einwandsfrei ist, als der Mann zunächst während zwei aufeinanderfolgender Fiebertage und dann zwei Wochen später nach vollständiger Entfieberung wieder untersucht wurde zu einer Zeit, wo sein Körpergewicht auf $49,4 \mathrm{~kg}$, also um $12,3 \mathrm{~kg}$ gesunken war. Das Fieber war lytisch abgefallen, und es waren nachher noch irreguläre Fieberbewegungen gefolgt. Afebril ist der Patient seit 20. Juli gewesen. In der Reconvalescenz wurde täglich nur ein Stuhl abgesetzt. Da sich gesteigerter Appetit einstellte, musste auch etwas mehr Nahrung zugeführt werden. Er erhielt in der fieberfreien Zeit:

2 Eier,

$750 \mathrm{ccm}$ Milch,

$250 \mathrm{ccm}$ Eiersuppe,

$250 \mathrm{ccm}$ Wein,

$150 \mathrm{ccm}$ Wasser. 
Tabelle XVI und XVII bieten eine Zusammenstellung sämmtlicher zur Beurtheilung des Versuches nöthigen Ergebnisse und Daten.

Tabelle XVI.

\begin{tabular}{c|c|c|c|c|c|c|c|c|c|c|c|c|c}
\hline $\begin{array}{c}\text { Versuchs- } \\
\text { tag }\end{array}$ & Datum & 8 & 10 & 12 & 2 & 4 & 6 & 8 & 10 & 12 & 2 & 4 & 6 \\
\hline 1. & $30 . / 5$. & 38,7 & 38,2 & 38,5 & 38,4 & 38,6 & 38,5 & 38,8 & 38,5 & 38,6 & 38,7 & 38,3 & 38,0 \\
2. & $31 . / 5$. & 37,8 & 38,2 & 38,5 & 38,4 & 38,6 & 38,5 & 38,3 & 38,1 & 37,8 & 38,0 & 37,9 & 37,7 \\
3. & $26 . / 6$. & 36,0 & 36,0 & 36,6 & 36,4 & 36,3 & 36,1 & 36,4 & 36,3 & 36,1 & 36,0 & 36,1 & 36,0 \\
4. & $\mathbf{2 7 . / 6 .}$ & 36,0 & 36,0 & 36,1 & 36,0 & 36,1 & 36,1 & 36,4 & 36,2 & 36,3 & 36,1 & 36,0 & 36,2
\end{tabular}

TABELLE XVII.

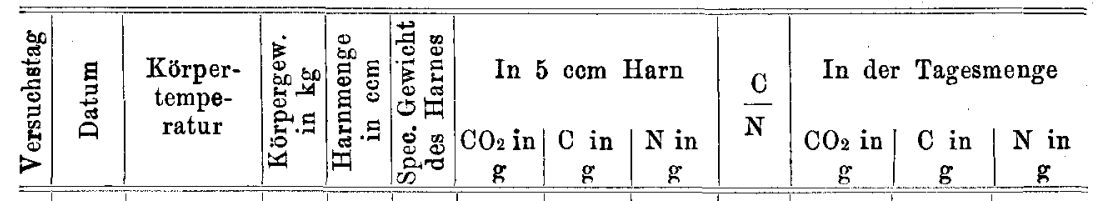

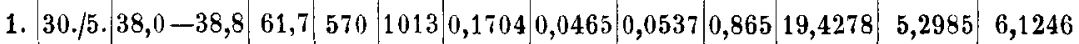

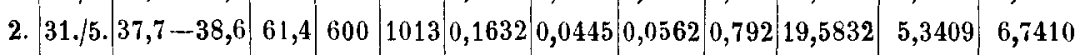

\begin{tabular}{l|l|l|l|l|l|l|l|l|l|l|l|l|l|l|l|l|l|l|l|}
3. & $26 . / 6$ & $36,0-36,6$ & 49,4 & 530 & 1020 & 0,2376 & 0,0648 & 0,0857 & 0,755 & 25,1818 & 6,8677 & 9,0895
\end{tabular}

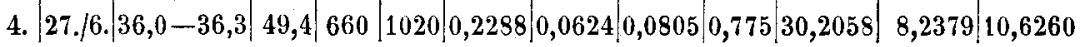

Der Umstand, dass die Einnahmen nicht die gleichen waren, konnte nach meinen früheren einschlägigen Untersuchungen keine in Betracht kommende Aenderung des Verhältnisses $\frac{C}{N}$ verursachen. Aber natürlich war infolge grösserer Einfuhr in der fieberfreien Zeit die Quantität des ausgeschiedenen Kohlenstoffes und Stickstoffes eine grössere. Die Relation $\frac{\mathrm{C}}{\mathrm{N}}$ war nach der Entfieberung merklich kleiner, so dass hier der Fieberharn gegenüber jenem der Reconvalescenzzeit relativ koblenstoffreicher erscheint. Der Unterschied des dritten Versuchstages gegen den ersten beträgt $0,11 \mathrm{~g}=13$ Proc.

In allen meinen Versuchen war der Unterschied, welchen das einzelne Individuum am Anfang und Ende darbot, erstlich vorhandenes oder nicht vorhandenes Fieber und weiteres eine gewisse Differenz des Körpergewichtes. Abgesehen von dem Typhusfalle (Versuch 8), zeigte die Relation $\frac{\mathrm{C}}{\mathrm{N}}$ in allen diesen Versuchen eine numerische Abweichung. Es ist kaum anzunehmen, dass diese Ab- 
weichung von der geringen constitutionellen Aenderung (Verminderung des Körpergewichtes, Aenderung des Eiweiss, Fettbestandes etc.) abhängt. Es liegt in der That näher, zu glauben, dass wirklich die Infecte hier das Maassgebende sind.

In allen meinen Versuchen geht aber zahlenmässig die Schwankung nicht über das Maass der Differenz hinaus, welche auch gesunde Menschen bei constantem Körpergewicht und Stickstoffgleichgewicht darbieten. Entgegen der Behauptung von $\mathrm{May}$, dass im Fieber ganz allgemein der Quotient $\frac{\mathrm{C}}{\mathrm{N}}$ gesteigert ist, beweisen meine am Menschen gemachten Versuche, dass ein solches eindentiges Verhalten keineswegs existirt. Für das Tuberculinfieber, für die Malaria des Menschen machte ich Beobachtungen, welche sich im Sinne der Annahme von May deuten lassen. Nach der Krise der Pneumonie geräth die Relation $\frac{\mathrm{C}}{\mathrm{N}}$ augenscheinlich in vom Temperaturablauf vollständig unabhängige, weil ganz entgegengesetzte Schwankungen. In dem Fall von Angina verbält sich die Sache eher umgekehrt, wie man nach May hätte erwarten müssen. Bei Typhus endlich fehlt, wie schon erwähnt, eine ersichtliche Aenderung. Ich behaupte auch keineswegs, dass etwa alle Fälle von Pneumonie, Typhus etc. des Menschen ganz dasselbe Verbalten des Quotienten $\frac{\mathrm{C}}{\mathrm{N}}$ zeigen müssten als in meinen doch relativ vereinzelten Beobachtungen; das aber muss ich aus meinen Untersuchungen doch mit aller Bestimmtheit entnehmen, dass bei den verschiedenen Fällen, beziehungsweise bei den verschiedenen Infecten kein eindeutiges Verhalten angenommen werden kann. Wenn man meiner Darlegung vielleicht die Schwierigkeit entgegen halten wollte, dass ich einmal fieberfreie Zeit ante und das andere Mal fieberfreie Zeit post febrem zum Vergleiche heranziehe, so muss ich mir diesen Einwand theilweise gefallen lassen. Diese Schwierigkeit ist nothwendig mit dem menschlichen Materiale gegeben. Wer an ein eindeutiges Verhalten der Relation $\frac{\mathrm{C}}{\mathrm{N}}$ glaubt, sollte einen solchen Einwand nicht erheben. Mindestens wird durch solche Bedenken keineswegs ein anderes Ergebniss meiner Versuche, welches von vornherein wahrscheinlich war, getroffen, dass die beobachteten Aenderungen des Quotienten $\frac{\mathrm{C}}{\mathrm{N}}$ keineswegs parallel gehen mit den Temperaturcurven und mit der Temperaturintensität. 


\section{Literatur.}

1. Ewald, Ueber den Kohlensăuregehalt des Harnes im Fieber. Reichert und Du Bois-Reymond's Archiv. 1873. S. 1.

2. A. Loew y, Stoffwechseluntersuchungen im Fieber und bei Lungenaffectionen. Virchow's Archiv Bd. CXXVI, 1891, S. 218.

3. R. May, Der Stoffwechsel im Fieber. Habilitationsschrift, München 1893 und Zeitschrift für Biologie Bd. XXX, 1894, S. 1.

4. Rubner, Ueber den Stoffverbrauch im hungernden Pflanzenfresser. Zeitschrift für Biologie Bd. XVII, S. 214.

5. Gottlieb, Experimentelle Untersuchung über die Wirkung temperaturherabsetzender Arzneimittel. Archiv f. experiment. Pathol. u. Parmakol. Bd. XXVI, 1890. S. 419.

6. Palmer, Ueber den Einfluss verschiedener Eingriffe auf die Körpertemperatur an Kaninchen und Hunden. Inaug.-Dissert. Strassburg 1886.

7. W. Scholz, Eine Methode zur Bestimmung des Kohlenstoffes organischer Substanzen auf nassem Wege und deren Anwendung auf den Harn. Centralblatt f. innere Med. 1897 . Nr. 15 u. 16.

8. F. Hirschfeld, Stoffwechseluntersuchungen bei Lungentuberculose nach Anwendung des Koch'schen Mittels. Berliner klin. Wochenschrift. 1891. Nr. 2. S. 29.

9. A. Loewy, Die Wirkung der Koch'schen Flüssigkeit auf den Stoffwechsel des Menschen. Berliner klin. Wochenschrift. 1891. Nr. 4. S. 93.

10. G. Klemperer, Die Einwirkung des Koch'schen Heilmittels auf den Stoffwechsel Tuberculöser. Dentsche med. Wochenschr. 1891. Nr. 15. S. 545. Graz, August 1897. 